\title{
TUDO O QUE É SÓLIDO DESMANCHA NO AR. OS MÉTODOS USADOS NAS PESQUISAS DE SISTEMAS DE INFORMAÇÃO NO BRASIL, UMA ANÁLISE DOS ÚLTIMOS CINCO ANOS DA ÁREA TEMÁTICA IMPACTOS SOCIOCUL- TURAIS DOS SISTEMAS DE INFORMAÇÃO NO ENANPAD
}

Everything that is solid disintegrates in the air: the used methods in the information system researchers in Brazil, an analysis of the last five years in the information system of the socio-cultural impacts thematic area in the ENAPAD

F. T. T. Ribas ${ }^{1}$

T. de Andrade ${ }^{2}$

M. L. Löbler $^{3}$

E. O.Simonetto ${ }^{4}$

\section{Resumo}

A área de Sistemas de Informação (SI) vem buscando sua consolidação para tornarse uma disciplina de referência, tornando-se necessário o rigor científico das pesquisas. Conseqüentemente há um crescente interesse na análise do rigor metodológico dos estudos

\footnotetext{
${ }^{1}$ Graduado em Administração na UNICRUZ, mestrando em Administração na UFSM. E-mail: fabiotolfo@hotmail. com

${ }^{2}$ Graduada em Administração na UNIFRA, mestranda em Administração na UFSM. E-mail: taisdeandrade@terra. com.br

${ }^{3}$ Doutor em Administração pela UFRGS, mestre em Administração pela UFSM, Professor do Curso de Mestrado em Administração da UFSM, Vice-Diretor do CCSH/UFSM. E-mail: lobler@ccsh.ufsm.br ${ }^{4}$ Doutor em Administração pela UFRGS, Professor do Departamento de Sistemas de Informação do Ceplan na Universidade do Estado de Santa Catarina (UDESC). E-mail: eosimonetto@ea.ufrgs.br
} 


\title{
TUDO O QUE É SÓLIDO...
}

nacionais e internacionais na área de SI. Assim, o objetivo deste artigo é apresentar um levantamento das metodologias e estratégias utilizadas nas publicações da área temática de Administração da Informação - Impactos Socioculturais dos Sistemas de Informação do ENANPAD, no período de 2003 a 2007. O método adotado nesta pesquisa foi o descritivo, caracterizando-se como uma pesquisa bibliográfica, em função do objetivo proposto. Verificouse, mediante a análise dos 52 artigos da área, que houve o predomínio dos estudos empíricos (92\%) em relação aos ensaios teóricos (8\%). Além disso, a abordagem qualitativa representou a preferência dos pesquisadores em 50\%, sendo o estudo de caso o tipo de pesquisa mais utilizado. Quanto à natureza, as pesquisas descritivas e exploratórias tiveram os percentuais mais relevantes, sendo o questionário o instrumento de coleta de dados preferencialmente adotado. Verificaram que poucos estudos utilizaram multimétodos em suas pesquisas, uma vez que estes possibilitam maior profundidade e complementaridade do estudo.

Palavras-chave: Sistemas de Informação; Administração da Informação; Produção Científica; Metodologias; Pesquisa.

\begin{abstract}
The Information System (IS) field is searching for its consolidation to become a reference subject, in order to that it is necessary a scientific rigor of the researches. Consequently, the interests in the analyses of the methodological rigor of the national and international studies of this field are growing. In this way, the aim of this paper is to present a survey of the methodologies and strategies used in publications of the Information Administration thematic area - Information System of the Socio cultural impacts of the ENANPAD, from 2003 to 2007. In order to get the proposed aim, the descriptive method (a bibliographic research) was adopted in this study. Through the analysis of 52 papers of the area, the predominance of the empirical studies (92\%) were found in comparison to theory ones (8\%). Furthermore, the qualitative approach represented the preference of the researchers in 50\%, and the case study was the most used way of research. In relation to the nature, the descriptive and exploratory researches had the most relevant percentages, and the questionnaire was the instrument of data collect preferentially adopted. It was verified that just few studies had used multimethods in their surveys and that they go deep and complement the study.
\end{abstract}

Keywords: Information Systems; Administration Information; Scientific Production; Methodologies; Search.

\section{Introdução}

A área de conhecimento Sistemas de Informação (SI) tem a interação da tecnologia de informação ( $\mathrm{Tl}$ ) com o ambiente organizacional como característica. O início dos anos 1990 representou um importante marco para a área, em razão dos avanços tecnológicos, popularização dos microcomputadores, Internet e possibilidade de novos negócios no ambiente virtual (LUNARDI, RIOS e MAÇADA, 2005).

A TI tem proporcionado diversas mudanças nas organizações, facilitando os processos empresariais, agregando estratégias proativas e contribuindo com a competitividade. Ela oferece as ferramentas que permitem às pessoas solucionar problemas cada vez mais complexos na organização, aproveitando oportunidades ou até mesmo a sobrevivência no mercado (TURBAN, RAINER e POTTER, 2005) 
A expansão da TI no meio organizacional é cada vez mais intensa. Assim, a área de Sistemas de Informação, ao longo dos últimos anos, tem dedicado esforço para tornar-se uma disciplina de referência no meio científico. Como afirmam Zimmer, Ferreira e Hoppen (2007), esta motivação deve-se à necessidade de afirmação da disciplina não somente como acessória no campo de administração, mas principalmente, como Ciência.

Diversos autores internacionais ressaltam a importância da consolidação da área, como Benbasat e Zmud (2003) e Baskerville e Myers (2002) que apontam a oportunidade para os pesquisadores de SI assumirem uma liderança mais visível e ativa dentro da comunidade científica, ressaltando a importância das referidas pesquisas para outras áreas de conhecimento. Os autores afirmam ainda, que as pesquisas em SI precisam ter qualidade e profundidade suficiente para que os estudantes e pesquisadores percebam a utilidade de tais investigações. Para tanto, as pesquisas devem ser abordadas no contexto da interdisciplinaridade, a fim de ultrapassar as fronteiras, para contribuir com diversas áreas do conhecimento por meio da intensificação de publicações, bem como pesquisas em conjunto com estudiosos de outras disciplinas.

Chen e Hirschheim (2004) afirmam existir um crescente interesse sobre a evolução das investigações de Sistemas de Informação no que diz respeito aos pressupostos filosóficos e metodológicos. A abordagem e análise das publicações empíricas na área de SI permitem uma investigação pluralista, que favorece a consolidação do corpo de conhecimentos da área.

Seguindo a tendência internacional, nos últimos anos, algumas publicações preocuparam-se em analisar o rigor metodológico das pesquisas nacionais na área de Sistemas de Informação, a fim de identificar a qualidade das publicações, validade dos instrumentos de coleta de dados, análise temática, sistematização de estratégias e métodos de pesquisa. Como afirmam Lunardi, Rios e Maçada (2005), identificar as metodologias adotadas permite estabelecer um panorama mais bem delineado da área, identificando a evolução dos estudos.

O artigo publicado por Hoppen (1998) identificou, por meio da análise de publicações da década de 1990, que a qualidade científica dos artigos ainda é baixa, em razão da não explicitação de como as teorias de base são operacionalizadas, da descrição incompleta dos procedimentos metodológicos e da não validação de pesquisa. A análise da produção científica em SI também foi abordada por Lunardi, Rios e Maçada (2005), que realizaram um estudo comparativo entre os artigos publicados no ENANPAD e nas principais revistas brasileiras de Administração, no período de 1997 a 2004. Nessa mesma linha, Oliveira, Maçada e Goldoni (2006) analisaram os aspectos relacionados ao planejamento, coleta e análise dos dados do método estudo de caso nas pesquisas em Sistemas de Informação publicados em periódicos e no ENANPAD.

Dessa forma, o objetivo deste artigo é apresentar um levantamento das metodologias e estratégias utilizadas nas publicações da área temática de Administração da Informação Impactos Socioculturais dos Sistemas de Informação do ENANPAD - no período 2003-2007. Para tanto, serão utilizados como elementos de análise a classificação dos artigos em qualitativos, quantitativos ou ambos, teóricos ou práticos, bem como a identificação do tipo e da natureza da pesquisa, além de instrumentos de coleta de dados. A referida análise permitirá identificar mais claramente as formas de investigação adotadas por essa área temática, bem como verificar se existe uma predominância de determinado método.

Cabe destacar uma parte do título deste trabalho, que faz referência ao livro de Berman (1986), como uma justificativa para esse levantamento, pois, como o autor afirma, "tudo o que é sólido se desmancha no ar", também se pode buscar entender mudanças numa determinada área do conhecimento desenvolvendo-se de forma dialética, ou seja, onde se destrói o antigo para se construir o novo, e nem mesmo a ciência, como se conhece, tem seu lugar garantido nas crenças de cada um de nós. Com base nessas premissas, é que se lança um olhar sobre 
a área temática de Administração da Informação - Impactos Socioculturais dos Sistemas de Informação - nos últimos anos, por meio da análise de suas publicações.

Na próxima seção, serão abordados alguns dos principais estudos publicados sobre análise de produção científica no Brasil e no exterior, nos últimos anos. Na seção 3, serão abordadas as principais metodologias de pesquisa utilizadas na área de SI. Na seção seguinte (seção 4), serão apresentados os aspectos metodológicos relacionados à análise aplicada neste estudo, para, posteriormente (seção 5), apresentarem-se os resultados encontrados. Por fim, na seção 6, serão apresentadas algumas considerações acerca do estudo realizado, limitações e sugestões para pesquisas futuras.

\section{Avaliação das Pesquisas nacionais e internacionais em SI}

A busca pela consolidação da área de Sistemas de Informação como disciplina científica tem fomentado inúmeros estudos com o objetivo de analisar as publicações a ela relacionadas. Essas pesquisas visam a identificar, entre outras estratégias, as áreas temáticas, metodologias utilizadas, predominância de métodos, paradigmas norteadores e a qualidade dos artigos.

Orlikowski e Baraudi (1991) realizaram uma pesquisa, na qual examinaram 155 artigos da área de SI na década de 1980, visando a identificar a gama de suposições filosóficas utilizadas nos estudos de fenômenos relacionados a sistemas de informação. Como resultados, constataram que a maioria dos artigos baseou-se no paradigma positivista, sendo a pesquisa survey e experimental os métodos predominantes.

Com a intenção de classificar a literatura de Sistemas de Informação, Barki et al. (1998 apud LUNARDI, RIOS e MAÇADA, 2005) classificaram diferentes tópicos de SI, sendo conhecido como o esquema de classificação MISQ (referindo-se ao artigo publicado na revista MIS Quarterly). Mais tarde, os mesmos autores revisaram a classificação original, atualizando as categorias propostas. O esquema levantou aproximadamente 1.100 palavras-chave, categorizando-as em nove níveis. Essa classificação foi utilizada por inúmeros estudos, como a pesquisa realizada no Brasil por Hoppen e Meirelles (2005).

Boudreau et al. (2001) realizaram um levantamento de 193 artigos dos cinco principais periódicos internacionais da área, a fim de identificar a validação dos instrumentos, pré-teste e confiabilidade. Segundo esses autores, os resultados apontaram que a maioria dos estudos publicados ainda não estão validando suficientemente seus instrumentos, se comparados com os resultados realizados por Straub em 1989, mesmo apresentando uma razoável evolução em relação à validação. Esses resultados comprovam que as pesquisas de SI, no exterior, ainda necessitam melhorar o rigor metodológico e a validação de instrumentos para tornar a área de Sistemas de Informação uma disciplina de referência.

Alguns desses estudos serviram de referência para as pesquisas realizadas posteriormente no Brasil. Seguindo uma linha próxima das investigações internacionais, Hoppen, Laponte e Moreau (1996) propuseram um guia para análise de artigos em SI, estabelecendo critérios rigorosos para a avaliação das pesquisas científicas realizadas no Brasil.

Posteriormente, em 1998, Hoppen realizou um estudo avaliando o estado da arte da área de SI no Brasil, com base na análise de 163 artigos publicados em revistas científicas e nos anais do ENANPAD no período de 1990 a 1997. As dimensões analisadas nos artigos foram a pesquisa empírica em relação à quantidade de ensaios teóricos, o mapeamento temático, as metodologias e estratégias das pesquisas em Sistemas de Informação e a análise da qualidade científica dos artigos. Quanto aos resultados da pesquisas, foram apontados que: $41 \%$ dos estudos eram ensaios teóricos, $46 \%$ das pesquisas empíricas eram do tipo survey e $26 \%$ eram estudos de caso, sendo 83\% exploratórias. No geral, detectou-se baixa qualidade, por causa da 
não explicitação da forma de operacionalização das teorias de base, descrição incompleta dos procedimentos metodológicos e a falta de validação dos instrumentos de pesquisa (HOPPEN, 1998).

Hoppen e Meirelles (2005) ampliaram a análise realizada anteriormente e investigaram 343 artigos publicados em revistas científicas de administração entre os anos de 1990 e 2003. A análise se concentrou nos temas abordados, nas estratégias e metodologias de pesquisa. Dessa forma, esses autores constataram que houve uma redução significativa dos ensaios teóricos de $41 \%$ para 9,4\%, no período 1990-1997, e uma redução das pesquisas exploratórias de $83 \%$ para $69 \%$. Verificaram também que os estudos de caso representaram o tipo de pesquisa predominante em $48 \%$, seguido das pesquisas survey, com $34 \%$. No entanto, como afirmam os autores, a melhoria verificada é importante, mas ainda não suficiente, pois os artigos analisados apresentaram ainda uma série de limitações metodológicas, revelando "dois problemas: a necessidade de intensificar o rigor científico no desenvolvimento da pesquisa e a falta de informações na apresentação dos resultados nos artigos científicos" (HOPPEN e MEIRELES, 2005, p. 32).

Com o objetivo de identificar os principais métodos utilizados na investigação dos tópicos e assuntos de pesquisa em SI, bem como a predominância de métodos relacionados ao tema dos estudos, Lunardi, Rios e Maçada (2005) analisaram 334 artigos dos anais do ENANPAD e das cinco principais revistas de Administração do Brasil (RAC, RAE, RAUSP, RAP e O\&S) no período de 1997 a 2004. Entre os resultados identificados, destaca-se o predomínio equilibrado dos estudos de caso (50,9\%) e pesquisas survey $(47,3 \%)$, corroborando os resultados encontrados por Hoppen e Meirelles (2005), que identificaram também o estudo de caso como tipo de pesquisa predominante. Além disso, os tópicos mais abordados foram os relacionados ao tema Administração de Sistemas de Informação.

Nessa linha de pesquisa, Oliveira, Maçada e Goldoni (2006) desenvolveram um framework, para analisar os aspectos relacionados ao planejamento, coleta e análise dos dados do método estudo de caso nas pesquisas em Sistemas de Informação. Os artigos analisados foram publicados no ENANPAD e nos principais periódicos nacionais da área (Qualis A) no período de 2003 a 2005. Os autores identificaram que a maioria dos artigos analisados não contemplou na íntegra os fatores que compõem o framework proposto. Isto não significa que as pesquisas não os consideraram. No entanto, não relataram o que foi realizado, o que dificulta o entendimento dos resultados obtidos e a sua aplicabilidade, dando pouca credibilidade aos resultados obtidos.

Por meio desse levantamento das pesquisas internacionais e nacionais relevantes à área, percebe-se que existem ainda deficiências em relação aos métodos utilizados. Ressalta-se que as metodologias de pesquisa contribuem para caracterizar o rigor científico das investigações, dando maior credibilidade à área de Sistemas de Informação.

\section{Métodos de pesquisa em SI}

Na área de Sistemas de Informação, alguns pesquisadores mostraram-se preocupados com o rigor metodológico dos estudos realizados. Nesse sentido, Hoppen, Lapointe e Moreau (1996) desenvolveram um guia de avaliação de pesquisas, detalhando cada critério utilizado, para verificar a qualidade e pertinência das estratégias utilizadas nos artigos. De forma mais específica, em relação ao tipo de pesquisa, Oliveira, Maçada e Goldoni (2006) desenvolveram um framework de avaliação de estudos de caso. Para esses autores, há uma grande necessidade de investigar os métodos de pesquisa em SI, a fim de consolidar a área como ciência 
independente. Inserido nessa estrutura de avaliação, há um construto denominado "Desenho de Pesquisa", que trabalha a forma estrutural dos artigos. A seguir será esclarecido cada item abordado no construto.

\subsection{Abordagem da pesquisa: qualitativa, quantitativa e qualitativa/ quantitativa}

A pesquisa qualitativa preocupa-se com um nível de realidade que não pode ser quantificada, trabalha com o universo de significados tais como: motivações, aspirações, crenças, valores e atitudes (MINAYO e SANCHES, 1993). Como afirmam Morgan e Smirich (1980), os métodos qualitativos podem valer-se de muitas técnicas para investigação, com diferentes tipos de suposições sobre ontologia e natureza humana, como, por exemplo, entrevistas, observação, análise de conteúdo e lingüística. Na pesquisa qualitativa, com a intenção de aprofundar os conhecimentos sobre determinado assunto, enfatiza-se a importância de compreender os processos nos quais os seres humanos criam a realidade.

Por outro lado, Richardson (1989) conceitua a pesquisa quantitativa como uma metodologia que impõe a estrutura predeterminada ao respondente, diminuindo a heterogeneidade da coleta dos dados e, assim, inferindo mais confiabilidade aos resultados. Conforme o referido autor, a pesquisa quantitativa trabalha com amostras mais amplas, fornecendo dados mais precisos em relação ao problema a ser estudado, sendo indicada quando já se tem mais informações sobre o problema a ser estudado.

Para Amaratunga etal. (2002), a escolha do mix das metodologias de pesquisa (qualitativa e quantitativa) é preferível porque diminui o risco de a pesquisa tornar-se inconsistente. O ideal é que os diferentes problemas sejam investigados de uma maneira complementar, a partir de visões tanto qualitativas como quantitativas. A comparação de resultados oriundos de investigações que utilizam métodos diferentes sobre os mesmos problemas pode contribuir para enriquecer sobremaneira o conhecimento sobre administração e organizações (VIEIRA, 2004).

Nesse sentido, Triviños (2007) afirma que a triangulação permite um aprofundamento sobre o tema investigado, detectando tanto a dimensão manifesta quanto a não-manifesta de um dado fenômeno social. A eficácia da triangulação refere-se à complementaridade entre os métodos qualitativo e quantitativo, ou seja, a fraqueza de um será compensada pela força do outro.

\subsection{Natureza da pesquisa}

Segundo Hair et al. (2005), os planos de pesquisa em administração podem ser agrupados em exploratórios, descritivos e causais.

Na pesquisa exploratória, trata-se de aprofundar conceitos preliminares, muitas vezes inéditos. O objetivo básico desse tipo de pesquisa é desenvolver hipóteses e proposições que irão redundar em pesquisas complementares (HOPPEN, LAPOINTE e MOREAU, 1996). Como afirma Richardson (1989), as investigações desta natureza objetivam a aproximação do pesquisador com o fenômeno, para que sejam identificadas as características e especificidades do tema a ser explorado.

Já uma pesquisa descritiva, segundo Trivinõs (2007), exige do pesquisador uma série de informações sobre o que ele deseja pesquisar. Tem como principal objetivo informar o pesquisador sobre situações, fatos, opiniões ou comportamentos da população analisada, 
buscando mapear a distribuição de um fenômeno.

A pesquisa causal é considerada aquela que testa uma teoria e suas relações causais. Para Hair et al. (2005), o conhecimento de relações causa e efeito fornece informações importantes para os tomadores de decisão, no entanto, os planos causais exigem uma execução precisa e complexa, além de um planejamento e controle rigoroso das variáveis.

\subsection{Tipos de pesquisa}

As pesquisas desenvolvidas em Sistemas de Informação, segundo Orlikowski e Baraudi (1991), utilizam predominantemente os métodos survey, experimental e qualitativo.

A pesquisa survey, segundo Pinsonneault e Kraemer (1993 apud HOPPEN, LAPOINTE e MOREAU, 1997), tem como característica descrever quantitativamente aspectos da população estudada, sendo a coleta de dados geralmente realizada a partir de questionários e uso de informações coletadas junto a uma amostra da população alvo. Segundo Gil (1999), as pesquisas desSe tipo caracterizam-se pela interrogação direta das pessoas, cujo comportamento se deseja conhecer, tendo como principais vantagens a economia, a rapidez e a quantificação.

Conforme Hoppen, Lapointe e Moreau (1997), pesquisa experimental é realizada num meio especialmente criado e controlado, que permite isolar as variáveis independentes e dependentes que serão estudadas. Ela implica numa manipulação dos sujeitos experimentais pelo pesquisador. Segundo Hair et al. (2005), as pesquisas experimentais podem ser classificadas em experimentos de laboratório, realizadas dentro de um ambiente artificial, ou de campo, conduzidas em um ambiente natural.

Outro tipo de pesquisa bastante utilizado é a pesquisa de cunho qualitativo, que se constitui numa importante contribuição à investigação das questões pertinentes à área da administração (GODOY, 1995). Além disso, Denzin e Lincoln (2006) afirmam que a pesquisa qualitativa envolve o estudo do uso e a coleta de uma variedade de materiais empíricos, que descrevem momentos e significados rotineiros e problemáticos na vida dos indivíduos. Nesse sentido, com o fim de especificar a abordagem qualitativa, Hoppen e Meirelles (2005) adotaram a classificação desse tipo de pesquisa em estudos de caso, pesquisa ação e outros (observação participante e o desenvolvimento de SI em laboratório).

Conforme Gil (1999), o estudo de caso é caracterizado pelo estudo profundo e exaustivo de um ou de poucos objetos, de maneira a permitir o seu conhecimento amplo e detalhado, tarefa praticamente impossível mediante os outros tipos de delineamento. Yin (2005) afirma ainda que os estudos de casos são aplicados na tentativa de explicar ligações causais em intervenções ou situações da vida real, que são complexas demais para tratamento por meio de estratégias experimentais ou de levantamento de dados (survey). Este autor salienta ainda que o estudo de caso é denominado múltiplo quando se refere a mais de um objeto de análise, sendo preferível ao estudo de caso único, pois os resultados emergem contrastes ou semelhanças, enriquecendo a pesquisa e favorecendo a replicação.

Na pesquisa-ação, segundo Hoppen, Lapointe e Moreau (1997), o pesquisador está envolvido simultaneamente na manipulação de certas variáveis e na avaliação dos fenômenos observados. Nesse tipo de pesquisa, a descrição dos procedimentos adotados para passar da ação (papel de agente) para a observação (papel de observador e analista) torna-se de suma importância. Devido ao caráter participativo, a pesquisa-ação tende a ser vista, em certos meios, como desprovida da objetividade que deve caracterizar os procedimentos científicos (GIL, 1999). 


\subsection{Instrumentos de coleta de dados}

Os pesquisadores buscam identificar os fenômenos por meio dos instrumentos de coleta de dados. Para Hoppen, Lapointe e Moreau (1997), na investigação científica, os instrumentos de pesquisa são utilizados para "ler a realidade". Na visão desses autores, nas Ciências Sociais, essa busca é ainda mais crítica, em razão dos fenômenos investigados estarem relacionados às percepções e comportamento dos indivíduos. Dessa forma, o pesquisador deve ser rigoroso quanto à adoção dos critérios de escolha, a fim de identificar características precisas do contexto estudado. Os resultados de uma pesquisa devem refletir a realidade, caso contrário, serão inconsistentes.

Os principais instrumentos de coletas de dados, conforme classificação de Sampaio e Perin (2006), são as entrevistas, questionários e análise documental, divididos nas categorias primários e secundários. Conforme Hair et al. (2005), se a natureza da pesquisa for exploratória, provavelmente o pesquisador irá coletar dados narrativos mediante o uso de entrevistas pessoais ou observação de comportamentos ou eventos. Ainda na visão desses autores, se o estudo for descritivo ou causal, o pesquisador preferencialmente irá utilizar os dados quantitativos mediante a aplicação de questionários.

Sergundo Hoppen, Lapointe e Moreau (1997), os principais meios de coleta utilizados na pesquisa survey são os questionários enviados pelo correio, o questionário utilizado em entrevista com a presença do entrevistador ou o questionário enviado por mídia eletrônica; na pesquisa experimental, a modalidade de coleta compreende o suporte empregado: o papel, o meio magnético e ótico, o meio audiovisual (fitas cassete de áudio e vídeo), além de entrevistas com os participantes, geralmente feitas ao final do processo experimental; e, nas pesquisas de caráter qualitativo, a coleta de dados dá-se por meio de entrevistas, análise de documentos e observação direta.

\section{Metodologia de análise}

A ABIC tem procurado imprimir uma estratégia voltada para qualidade em todo o parque iEste estudo adotou o método descritivo de pesquisa, pois foram analisadas as metodologias e estratégias utilizadas nas publicações da área temática Administração da Informação - Impactos Socioculturais dos Sistemas de Informação. Para Hair et al. (2005), os estudos descritivos são estruturados e especificamente criados para medir as características descritas em uma questão de pesquisa, ou seja, é a mensuração de um evento ou atividade. Além disso, segundo Gil (1999), os estudos descritivos objetivam a descrição das características de determinada população ou fenômeno e também o estabelecimento de relações entre variáveis.

Quanto aos procedimentos técnicos, caracterizou-se como uma pesquisa bibliográfica, baseada nos artigos publicados no Encontro Nacional da ANPAD, no período de 2003 a 2007. Optou-se pela escolha das publicações do ENANPAD em função de sua importância no cenário nacional, sendo o principal veículo de divulgação científica do que se desenvolve na área de SI na atualidade (LUNARDI, RIOS e MAÇADA, 2005).

Foram analisados 52 artigos da área temática Impactos Socioculturais, que abordam assuntos relacionados aos impactos do SI nos indivíduos, organizações e sociedade, no que diz respeito aos aspectos comportamentais, culturais e ambientais. Desses artigos, quatro foram classificados como teóricos e 48 como empíricos. Dessa forma, a análise dos métodos e instrumentos utilizados foi realizada somente nos trabalhos empíricos.

A análise dos tipos baseou-se no guia de avaliação de artigos de pesquisa, proposto por Hoppen, Lapointe e Moreau (1996), e na adaptação do estudo de Hoppen e Meirelles (2005), 
que classificam os estudos em: estudos de caso, pesquisa survey e estudos experimentais. Quanto à natureza da pesquisa, seguiu-se a classificação proposta por Hair et al. (2005), que classificaram as pesquisas em exploratória, descritiva e causal. Em relação aos instrumentos de coleta de dados, optou-se em adaptar a classificação proposta por Sampaio e Perin (2006), subdividindo as categorias em: entrevistas, questionários, dados secundários, primários e secundários e multimétodos (pesquisa qualitativa e quantitativa).

Dessa forma, conforme o Quadro 1, a análise dos referidos artigos baseou-se na adaptação dos modelos dos referidos autores.

\begin{tabular}{|c|c|}
\hline \multicolumn{2}{|r|}{ Classificação de metodologias de pesquisa } \\
\hline Abordagem & $\begin{array}{l}\text { Qualitativa } \\
\text { Quantitativa } \\
\text { Qualitativa/quantitativa }\end{array}$ \\
\hline \multirow{3}{*}{ Tipo de pesquisa } & $\begin{array}{l}\text { Survey } \\
\text { Experimental }\end{array}$ \\
\hline & Estudo de caso único (qualitativa) \\
\hline & $\begin{array}{l}\text { Estudo de caso múltiplo (qualitativa) } \\
\text { Pesquisa ação (qualitativa) }\end{array}$ \\
\hline \multirow{3}{*}{$\begin{array}{l}\text { Natureza da } \\
\text { pesquisa }\end{array}$} & Exploratória \\
\hline & Descritiva \\
\hline & Causal \\
\hline \multirow[t]{2}{*}{$\begin{array}{l}\text { Instrumento de } \\
\text { coleta de dados }\end{array}$} & $\begin{array}{l}\text { Entrevista } \\
\text { Questionário } \\
\text { Multi métodos }\end{array}$ \\
\hline & $\begin{array}{l}\text { Secundários } \\
\text { Primá rios e Secundários }\end{array}$ \\
\hline
\end{tabular}

Fonte: elaborado com base nos estudos de Hoppen, Lapointe e Moreau (1996); Hoppen e Meirelles (2005); Hair et al. (2005) e Sampaio e Perin (2006).

Quadro 1 - Classificação de metodologias de pesquisa.

Os 52 artigos selecionados foram analisados e classificados por dois pesquisadores, conforme os critérios já referidos. Inicialmente, foram analisados 10 artigos em conjunto, a fim de garantir maior padronização e consistência nos critérios de classificação. Posteriormente, cada pesquisador trabalhou de forma independente e, ao fim da classificação individual, os resultados foram confrontados. No total, sete $(13,4 \%)$ artigos apresentaram divergência de classificação entre os pesquisadores, por causa da utilização de instrumentos de coleta de dados diversos.

Realizada a descrição da metodologia adaptada para a referida análise dos artigos, apresentam-se, na seção seguinte, os resultados encontrados.

\section{Resultados}

A descrição dos resultados foi efetuada por meio da análise de 52 artigos publicados no ENANPAD, no período de 2003 a 2007, na área Impactos Socioculturais do SI. Convém ressaltar que, na análise, houve certa dificuldade na identificação de qual metodologia foi usada nas pesquisas de alguns artigos. Nesses estudos, não foram explicitados, de forma clara, 
os métodos utilizados e também não foram descritos os detalhes relativos aos procedimentos adotados na pesquisa.

Como afirmam Hoppen, Lapointe e Moreau (1996, p. 3), "cada pesquisador, que fez o exercício de avaliar formalmente o conteúdo de um artigo científico de pesquisa em SI, sabe que grande parte dos artigos publicados não anuncia de modo explícito os métodos de pesquisa utilizados". Em vários casos, autores negligenciam certos detalhes essenciais para a avaliação da pesquisa e torna-se difícil a apreciação do estudo feito. Assim, identificou-se, em alguns artigos, que a especificação do método utilizado não estava descrita na metodologia de pesquisa, estando implícita em outras seções do artigo, como na análise dos resultados.

\subsection{Ensaios teóricos e pesquisas empíricas}

Inicialmente é importante destacar que, do total de artigos analisados, apenas quatro (ou 8\%) foram classificados como ensaios teóricos e 48 (ou 92\%), como pesquisa empírica, conforme a Tabela 1.

Esse resultado mostra a mesma tendência da pesquisa realizada por Claver, Gonzales e Lopis (1999), em um estudo realizado por meio da análise de artigos publicados nos principais periódicos internacionais, no período de 1981 a 1997, nos qual houve um aumento no número de artigos empíricos em relação aos teóricos. Segundo esses autores, isso indica que os estudos teóricos eram mais satisfatórios no passado e que, atualmente, a popularidade dos estudos empíricos é maior, pois visam a provar teorias que já existem na prática ou construir teorias baseadas em fatos. Resultado semelhante também foi encontrado no levantamento realizado por Hoppen e Meirelles (2005), os quais destacaram que os artigos teóricos representavam apenas 9,4\% dos artigos analisados no período 1990-2003 nas publicações nacionais.

Conforme Hoppen e Meirelles (2005), a queda acentuada no número de ensaios teóricos revela que a pesquisa empírica está em franco desenvolvimento na área e denota uma consolidação da produção de conhecimento local em SI. Nesse sentido, Lunardi, Rios e Maçada (2005) identificaram, em seu estudo, que os programas de pós-graduação em Administração foram os precursores da tentativa de consolidação da área de SI no Brasil, responsáveis por mais da metade das publicações, sendo predominante os estudos empíricos.

Tabela 1 - Classificação dos artigos na área Impactos Socioculturais dos Sistemas de Informação

\begin{tabular}{lcccc|c|c|c}
\hline $\begin{array}{c}\text { Classificação dos } \\
\text { trabalhos }\end{array}$ & 2003 & 2004 & 2005 & 2006 & 2007 & Total & $\%$ \\
Pesquisa empírica & 10 & 9 & 10 & 10 & 9 & 48 & $92 \%$ \\
\hline Ensaio teórico & 0 & 1 & 0 & 1 & 2 & 4 & $8 \%$ \\
\hline
\end{tabular}

\subsection{Abordagem da pesquisa}

$\mathrm{Na}$ análise realizada nos estudos em relação à abordagem da pesquisa, houve a predominância da pesquisa qualitativa. No total, a metodologia qualitativa representou $50 \%$; a quantitativa, 38\% e a mista (qualitativa e quantitativa) atingiu 13\%, conforme a Tabela 2.

Convém ressaltar que, na década de 1990, nas pesquisas da área Tecnologia da 
Informação, predominavam pontos de vista positivistas, pelo uso intenso de metodologias quantitativas (GALLIVAN, 1997 apud TEIXEIRA JÚNIOR, 2001). Percebeu-se esta predominância no estudo realizado por Hoppen (1998), no qual a pesquisa survey representou $46 \%$ dos estudos analisados. No entanto, no levantamento realizado por Teixeira Júnior (2001), no qual o autor analisa publicações na área de SI no período de 1999 a 2001, houve a predominância dos estudos qualitativos em 55,3\% dos artigos. Da mesma forma, o presente estudo apresentou resultados semelhantes à pesquisa do referido autor, contrariando a tendência dos anos 1990.

A abordagem qualitativa, na área de SI, busca uma compreensão a partir da interação dos indivíduos com as tecnologias de informação, bem como os impactos que estas irão causar nas rotinas de trabalho, no comportamento e no ambiente organizacional como um todo. Por outro lado, a pesquisa quantitativa procura mensurar os dados reais, analisá-los estatisticamente, relacionando-os com as tecnologias de informação.

Tabela 2 - Abordagem da pesquisa

\begin{tabular}{cc|c|c|c|ccc|}
\hline Abordagem & 2003 & 2004 & 2005 & 2006 & 2007 & Total & $\%$ \\
\hline Qualitativa & 5 & 3 & 7 & 5 & 4 & 24 & $50 \%$ \\
Quantitativa & 3 & 5 & 2 & 4 & 4 & 18 & $38 \%$ \\
Qualitativa e quantitativa & 2 & 1 & 1 & 1 & 1 & 6 & $13 \%$ \\
\hline Total & 10 & 9 & 10 & 10 & 9 & 48 & $100 \%$
\end{tabular}

\subsection{Tipos de pesquisa}

A Tabela 3 apresenta o resultado do levantamento metodológico realizado sobre os tipos de pesquisa. Percebe-se a predominância do estudo de caso único (48\%), seguido da pesquisa survey (27\%). Por outro lado, a pesquisa-ação (4\%) e a experimental ( $8 \%$ ) foram pouco utilizadas no período compreendido do estudo. Cabe ressaltar que não houve uma evolução da utilização de determinado método ao longo dos anos analisados, mantendo-se constante a média de trabalhos que utilizam referida tipologia.

Em relação ao estudo de caso, tipo de pesquisa predominante, identificou-se que os estudos de caso único sobressaíram-se em relação aos estudos de caso múltiplo. Conforme Yin (2005), o estudo de caso é uma investigação empírica que aplicada na tentativa de explicar ligações causais em situações da vida real, pois estas são complexas demais para o tratamento por meio de estratégias experimentais ou de levantamento de dados. Dessa forma, o estudo de caso predominou nas investigações da área temática Impactos Socioculturais dos Sistemas de Informação, que aborda, entre outros temas, os assuntos relacionados aos impactos do SI no comportamento dos indivíduos, cultura das organizações e sociedade, com a intenção de aprofundar o conhecimento de determinado objeto.

Convém ressaltar a visão de Weber (1993, p. 130) quanto ao caráter qualitativo das investigações sociais: "Para o conhecimento das condições concretas dos fenômenos históricos, as leis mais gerais são, freqüentemente as menos valiosas, por serem as mais vazias de conteúdo". Na percepção desse autor, a importância atribuída à investigação dos fenômenos sociais e dos conhecimentos produzidos nas Ciências Sociais não passam pelo roteiro da generalização, e sim pela compreensão do sentido das ações das pessoas, a fim de descobrir por que agem de determinada maneira. 
Constata-se, dessa forma, que há uma preocupação dos pesquisadores da área com o estudo detalhado de determinado objeto de estudo. Como afirmam Oliveira, Maçada e Goldoni (2006), a predominância do estudo de caso pode representar que a área de Sistemas de Informação no Brasil está buscando um melhor entendimento dos fenômenos para, posteriormente, passar para a construção de teorias a partir da prática ao utilizar outros métodos como, por exemplo, a survey. Essa tendência é evidenciada neste estudo, visto que a pesquisa survey foi o segundo tipo predominante.

O baixo número de pesquisas experimentais, apenas quatro estudos no período de 2003 a 2007, está associado, principalmente, à dificuldade da área em obter uma estrutura adequada e recursos suficientes (CLAVER, GONZALEZ e LOPIS, 1999). Andrade (2004) afirma que, nas pesquisas experimentais, os fenômenos são estudados nas condições determinadas pelo experimentador, ou seja, requerendo a manipulação e o controle das variáveis. Por outro lado, nas Ciências Sociais, nem sempre é possível realizar pesquisas com controle rigoroso de variáveis, por isso são chamadas de pesquisas "quase experimentais". Dessa forma, utiliza-se o método de observação, no qual os fenômenos são estudados como se apresentam, pois o objeto de estudo é geralmente o comportamento humano.

Os resultados encontrados seguem a mesma tendência dos resultados descritos por Hoppen e Meirelles (2005), nos quais o estudo de caso predominou em $48 \%$ e a pesquisa survey, em 34\%. Resultados semelhantes também foram encontrados por Lunardi, Rios e Maçada (2005), nos quais os estudos de caso predominaram, com 50,9\%, seguidos dos tipo survey, com 47,3\%, no período de 1997-2004.

Tabela 3 - Tipos de pesquisa

\begin{tabular}{|c|c|c|c|c|c|c|c|}
\hline Tipos de pesquisa & 2003 & 2004 & 2005 & 2006 & 2007 & Total & $\overline{\%}$ \\
\hline Survey & 1 & 4 & 1 & 4 & 3 & 13 & $27 \%$ \\
\hline Experimental & 0 & 1 & 2 & 0 & 1 & 4 & $8 \%$ \\
\hline Estudo de caso único (qualitativa) & 9 & 4 & 3 & 3 & 4 & 23 & $48 \%$ \\
\hline Estudo de caso múltiplo (qualitativa) & 0 & 0 & 2 & 3 & 1 & 6 & $13 \%$ \\
\hline Pesquisa ação (qualitativa) & 0 & 0 & 2 & 0 & 0 & 2 & $4 \%$ \\
\hline Total & 10 & 9 & 10 & 10 & 9 & 48 & $100 \%$ \\
\hline
\end{tabular}

\subsection{Natureza da pesquisa}

Nos artigos empíricos investigados, em relação à natureza da pesquisa, predominaram os estudos exploratórios (50\%) e a pesquisa descritiva (48\%), em percentuais muito semelhantes, conforme se verifica na Tabela 4. Hoppen (1998), ao descrever o cenário das pesquisas em SI nos anos 1990, destacou que havia a predominância das pesquisas exploratórias em mais de $80 \%$ dos estudos analisados; no entanto, em 2005, mediante análise dos artigos no período de 1990 a 2003, constatou que houve uma redução dos estudos exploratórios para 69\%, sendo que, neste trabalho, a tendência é confirmada. 
Tabela 4 - Natureza da pesquisa

\begin{tabular}{ccc|c|cccc}
\hline Natureza da pesquisa & $\mathbf{2 0 0 3}$ & $\mathbf{2 0 0 4}$ & $\mathbf{2 0 0 5}$ & $\mathbf{2 0 0 6}$ & $\mathbf{2 0 0 7}$ & total & $\%$ \\
\hline Exploratória & 7 & 2 & 6 & 4 & 5 & 24 & $50 \%$ \\
Descritiva & 3 & 7 & 4 & 5 & 4 & 23 & $48 \%$ \\
Causal & 0 & 0 & 0 & 1 & 0 & 1 & $2 \%$ \\
\hline Total & 10 & 9 & 10 & 10 & 9 & 48 & $100 \%$
\end{tabular}

A Figura 1 demonstra a aplicação do tipo de método e natureza da pesquisa utilizada nos estudos analisados. Cabe ressaltar a predominância dos estudos exploratórios e descritivos nos estudos de caso e pesquisa survey. Percebe-se também que a pesquisa causal foi utilizada em apenas um estudo, corroborando com os resultados encontrados por Lunardi, Rios e Maçada (2005), que não detectaram nenhum estudo causal, demonstrando a predominância absoluta de estratégias descritivas e exploratórias. Além disso, a pouca incidência de estudos causais pode ser explicada pelas exigências complexas e precisas, bem como planejamento e controle rigoroso das variáveis que este tipo de plano exige, dificultando as pesquisas nessa área.

Nos estudos experimentais e pesquisa-ação, predominou a estratégia exploratória, revelando que essas investigações objetivam descobrir aspectos desconhecidos ou pouco explorados. No entanto, em razão do número reduzido de pesquisas dessa natureza, quatro estudos experimentais e dois caracterizados como pesquisa-ação, a análise desses casos é bastante restrita.

A pesquisa exploratória, segundo Andrade (2004), proporciona maiores informações sobre o assunto investigado, orientando a descoberta de um novo tipo de enfoque. Hoopen, Lapointe e Moreau (1996) ratificam, ainda, que esse tipo de pesquisa aprofunda conceitos preliminares, muitas vezes inéditos. Por outro lado, a pesquisa descritiva visa a descrever as características de determinada população, ou fenômeno, bem como estabelecer relação entre variáveis (GIL, 1999). Evidencia-se, dessa forma, uma evolução parcial da área, pois a preocupação pela descoberta de novas temáticas está sendo substituída gradualmente pela confirmação dos dados já explorados. Essa evidência é comprovada também nos estudos anteriores, realizados por Hoppen e Meirelles (2005) e Lunardi, Rios e Maçada (2005), que apontaram uma tendência decrescente das pesquisas exploratórias e crescentes para pesquisas descritivas.

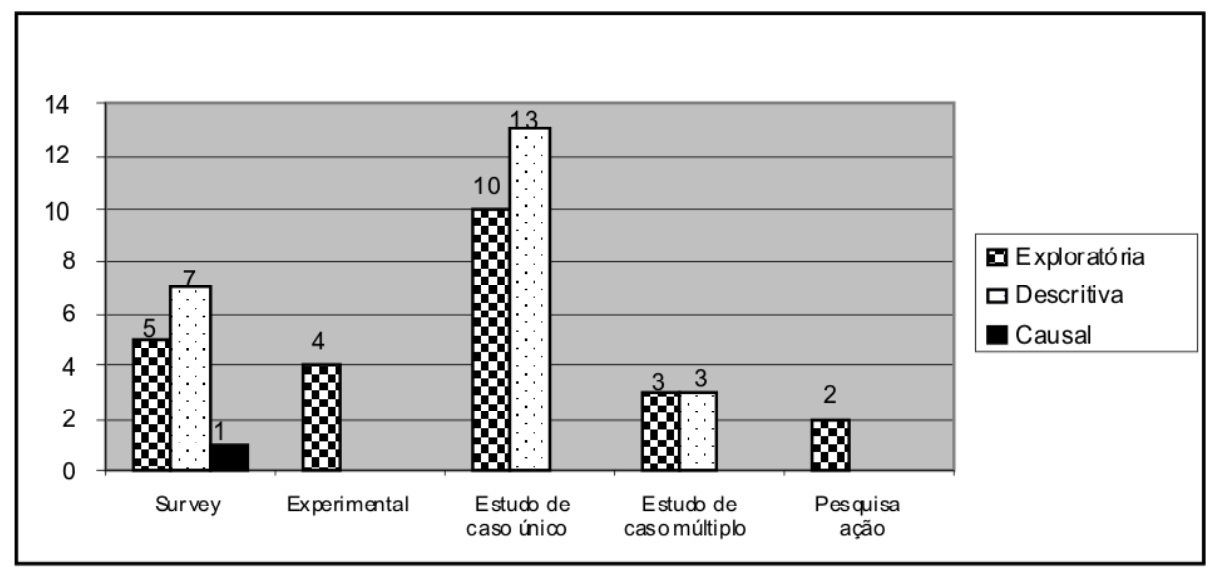

Figura 1 - Cruzamento do tipo de método e natureza da pesquisa. 
Evidenciou-se, neste trabalho, a predominância das entrevistas (27\%) e dos questionários (29\%) como instrumentos de coleta de dados, conforme ilustrado na Tabela 5. Conforme os autores Amaratunga et al. (2002), Vieira (2004) e Triviños (2007), citados anteriormente, a escolha das metodologias qualitativas em conjunto com as quantitativas é preferível à escolha isolada de uma delas, pois a eficácia da triangulação refere-se à complementaridade entre os métodos, garantindo maior profundidade do estudo. Neste trabalho, as práticas multimétodos (quantitativas e qualitativas) representaram 13\% dos instrumentos utilizados pelos pesquisadores, contrariamente às práticas sugeridas pelos referidos autores. A tendência para consolidação da área poderá caracterizar-se pelo aumento da utilização de multimétodos em detrimento de um único instrumento de coleta de dados.

Os instrumentos de coleta de dados primários e secundários, como, por exemplo, utilização de entrevistas e análise de documentos da empresa, apresentaram um índice de utilização de $29 \%$, o que demonstra a preocupação dos pesquisadores em fundamentar os dados obtidos por meio de diferentes fontes de evidência. Segundo Hoppen e Meirelles (2005), a combinação dos dados primários e secundários favorece a triangulação dos dados.

Os dados secundários representaram apenas $2 \%$ dos instrumentos utilizados. Conforme Hoppen e Meirelles (2005), o pequeno número de pesquisas baseadas exclusivamente em dados secundários indica a pouca disponibilidade ou confiança na utilização de outras fontes de consulta, como banco de dados e publicações legais.

Tabela 5 - Instrumento de coleta de dados

\begin{tabular}{|cc|c|cc|c|cc|}
\hline Instrumento de coleta de dados & 2003 & 2004 & 2005 & 2006 & $\mathbf{2 0 0 7}$ & Total & $\%$ \\
\hline Entrevista & 3 & 2 & 3 & 4 & 1 & 13 & $27 \%$ \\
Questionário & 2 & 5 & 0 & 4 & 3 & 14 & $29 \%$ \\
Multimétodos & 2 & 1 & 1 & 1 & 1 & 6 & $13 \%$ \\
\hline Secundários & 0 & 0 & 1 & 0 & 0 & 1 & $2 \%$ \\
Primários e Secundários & 3 & 1 & 5 & 1 & 4 & 14 & $29 \%$ \\
\hline Total & 10 & 9 & 10 & 10 & 9 & 48 & $100 \%$ \\
\hline
\end{tabular}

Analisando-se as informações referentes aos instrumentos de coleta de dados e tipo de pesquisa, conforme Figura 2, verificou-se que, na pesquisa survey, houve o predomínio do uso de questionário (76\%). O predomínio de questionários nesse tipo de pesquisa ocorre principalmente pela facilidade e conveniência, em razão da grande amostra de indivíduos. Além disso, uma boa pesquisa survey exige bons questionários para garantir a precisão dos dados coletados (HAIR et al., 2005). Esses resultados estão de acordo com a pesquisa realizada por Hoppen e Meirelles (2005), na qual houve o predomínio dos dados primários (questionários).

Em relação ao estudo de caso único, as entrevistas representaram 39\% dos instrumentos de coleta de dados utilizados. Essa predominância deve-se principalmente ao caráter qualitativo dos estudos de caso, nos quais as entrevistas caracterizam-se como os instrumentos mais adequados. Conforme Godoy (1995), no estudo de caso, a principal finalidade é a análise em profundidade da natureza das interações de determinado fenômeno. Dessa forma, utilizam-se os métodos entrevista e a observação.

Nos tipos de pesquisa estudo de caso múltiplo, experimental e pesquisaação, por causa do número reduzido de estudos, não foi possível inferir a predominância de determinado instrumento de coleta de dados. 


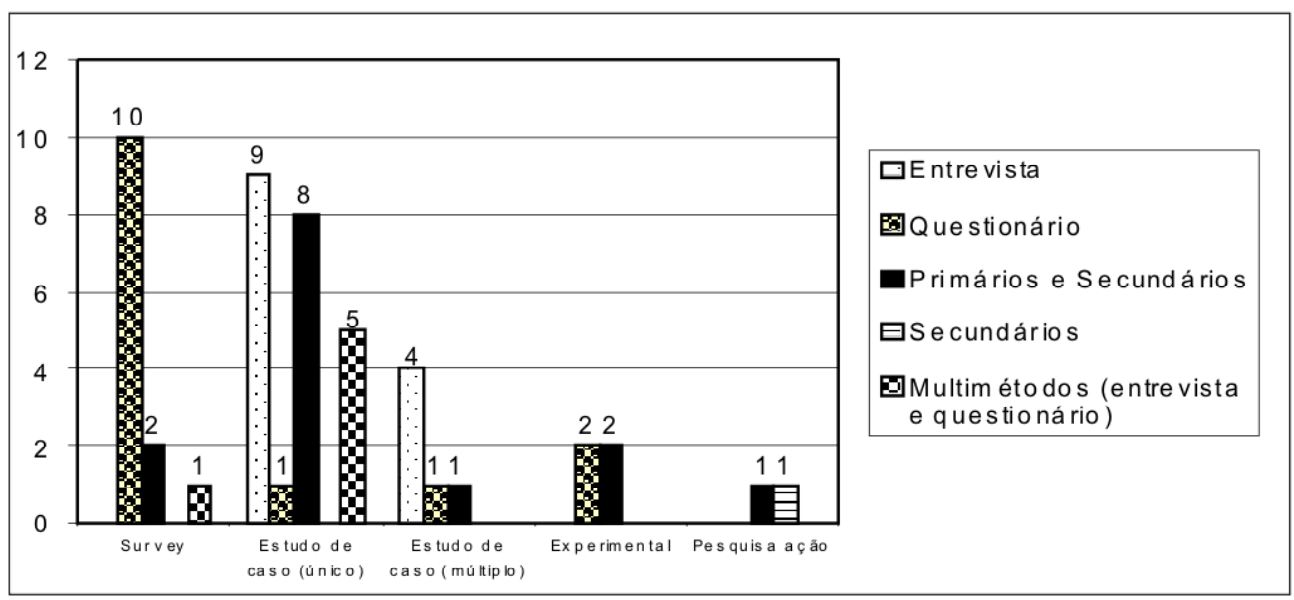

Figura 2 - Cruzamento instrumento de coleta de dados e tipo de pesquisa.

Sendo assim, os resultados obtidos na referida área temática de SI, no período de 2003 a 2007, evidenciaram a predominância de métodos qualitativos (estudos de caso), principalmente os descritivos, seguidos dos exploratórios, com um pequeno percentual de diferença. Embora os instrumentos de coleta de dados primários e secundários tenham predominado na referida análise, demonstrando a preocupação dos pesquisadores em relação à cientificidade pelo uso de múltiplas fontes de evidência, percebe-se que a triangulação metodológica (dados qualitativos e quantitativos) ainda tem sido pouco adotada.

Convém ressaltar, ainda, que os resultados encontrados nas pesquisas realizadas por Hoppen e Meirelles (2005) e Lunardi, Rios e Maçada (2005), em que esses autores analisaram artigos da área de SI de maneira global nas principais revistas científicas e ENANPAD, foram semelhantes em relação aos aspectos metodológicos predominantes nos artigos da área Impactos Socioculturais de SI.

\section{Considerações finais}

A análise dos artigos publicados na área temática de Administração da Informação - Impactos Socioculturais dos Sistemas de Informação do ENANPAD, no período de 2003 a 2007, permitiu apresentar um panorama das metodologias e estratégias utilizadas nos estudos. Percebe-se que a área de SI está em busca da consolidação como disciplina de referência nos estudos em Administração, sendo o rigor metodológico um fator determinante para garantir a cientificidade das pesquisas.

Os artigos analisados apresentaram algumas limitações quanto à especificação dos métodos e, em alguns casos, estavam descritos em outras seções dos artigos e não na metodologia do estudo. A preocupação estrutural, no caso do desenho de pesquisa tratado neste artigo, contribui para o rigor científico no desenvolvimento da pesquisa bem como a credibilidade dos estudos. Convém ressaltar que as pesquisas internacionais e nacionais têm demonstrado interesse pela precisão metodológica e pela abordagem das estratégias de pesquisas utilizadas nos estudos da área (CHEN E HIRSCHHEIM, 2004; HOPPEN E MEIRELLES, 2005; LUNARDI, RIOS E MAÇADA, 2005).

No presente estudo, houve a predominância dos estudos empíricos (92\%), evidenciando a busca pelo desenvolvimento e consolidação da produção científica da área. Em relação à abordagem de pesquisa, constatou-se que a pesquisa qualitativa $(50 \%)$, preponderante nos estudos, revela a busca por uma maior compreensão da interação dos indivíduos com 
a tecnologia da informação, principal foco da área temática analisada. Quanto ao tipo de pesquisa, predominou o estudo de caso único (48\%), enfatizando o aprofundamento dos conhecimentos de determinado objeto; no caso, o impacto e relação da TI com os indivíduos, empresa e ambiente.

Referindo-se à natureza da pesquisa, prevaleceu a pesquisa exploratória (50\%). Cabe comentar que os estudos exploratórios, em pesquisas anteriores, apresentaram índices decrescentes. Hoppen (1998) afirmou que as pesquisas dessa natureza, na década de 1990, representavam $80 \%$ e, na pesquisa posterior, realizada no ano de 2005, esse índice caiu para $69 \%$. Isso demonstra que as pesquisas exploratórias estão sendo substituídas gradualmente pelas descritivas, que, neste estudo, representaram (48\%). Essa tendência evidencia que os estudos na área de SI estão evoluindo de uma abordagem em que se investigam fenômenos ou conceitos inéditos para uma ênfase na descrição e relação entre variáveis, demonstrando a continuidade das etapas necessárias para a legitimação da área.

Quanto aos instrumentos de coleta de dados, evidenciou-se a predominância dos questionários (29\%) e dos dados primários e secundários (29\%), seguidos pelas entrevistas (27\%). A escolha da complementaridade dos instrumentos para coletar os dados é preferível à escolha isolada de um deles, pois muitos autores sugerem que a triangulação é eficaz, garantindo a profundidade do estudo. No entanto, percebeu-se, no levantamento realizado, que o uso de multimétodos (13\%) ainda é pouco utilizado nas pesquisas da área. Convém ressaltar que o objetivo deste estudo foi o de identificar as estratégias e métodos utilizados, sem avaliar a qualidade e a pertinência de sua utilização. Dessa forma, pode-se inferir somente que as práticas adotadas nos estudos analisados são contrárias às sugestões quanto às práticas da triangulação metodológica, podendo limitar os resultados da pesquisa.

Em relação aos tipos de pesquisa, percebeu-se que, na pesquisa survey, predominou a abordagem descritiva e exploratória, sendo o questionário o principal instrumento, por possibilitar a amplitude na coleta dos dados. No estudo de caso, houve o predomínio dos estudos descritivos e exploratórios e o principal instrumento foi a entrevista, em razão do caráter qualitativo dos estudos, enfatizando a profundidade de interação com o fenômeno. Nos estudos experimentais e pesquisa-ação, devido ao número reduzido de estudos, não foi possível inferir a predominância da natureza e instrumento de coleta de dados utilizados.

Diante dos resultados obtidos, o predomínio dos estudos qualitativos nos estudos da área demonstra um interesse dos pesquisadores na compreensão e aprofundamento de determinado fenômeno, focando as questões organizacionais e comportamentais em relação à utilização das tecnologias de informação. Isso também foi evidenciado nos estudos de Hoppen e Meirelles (2005) e de Lunardi, Rios e Maçada (2005), demonstrando a predominância dos estudos de caso na área de SI de forma global. No entanto, cabe ressaltar a necessidade de continuidade e análise do rigor científico no desenvolvimento das pesquisas, bem como a ampliação dos estudos em SI, pois a área está em busca de consolidação.

Ressalta-se que, como toda pesquisa, devem ser considerados os limites do estudo, principalmente quanto a sua abrangência - área temática de Administração da Informação Impactos Socioculturais de SI e análise metodológica restrita ao desenho estrutural. Dessa forma, a fim de complementar este estudo, sugere-se a análise em relação à adequação metodológica, aos paradigmas predominantes, à qualidade dos artigos e à análise temática. Além disso, podemse analisar, com maior profundidade, as pesquisas qualitativas, principalmente o estudo de caso, no âmbito dos principais periódicos nacionais e ENANPAD num maior período de tempo, para que seja traçado um panorama da evolução desses estudos na área de SI. 


\section{Referências bibliográficas}

AMARATUNGA, D. et al. Quantitative and qualitative research in the built environment: application of "mixed" research approach. Work Study, v. 51, n.1, p. 17-31, 2002.

ANDRADE, M. M. Como preparar trabalhos para cursos de Pós-Graduação: noções práticas. 6. ed. São Paulo: Atlas, 2004.

BASKERVILLE, R.; MYERS, M. Information systems as a reference discipline. MIS Quarterly, v. 26, n. 1, p. 114-118, 2002.

BENBASAT, I.; ZMUD, R.W. The identity crisis within the IS discipline: defining and communicating the discipline's core properties. MIS Quarterly, v. 27, p. 183-194, 2003.

BERMAN, M. Tudo que é sólido desmancha no ar: a aventura da modernidade. Rio de Janeiro: Cia. das Letras, 1986.

BOUDREAU, M-C. et al. Validation in information systems research: a state-of-the-art assessment. MIS Quarterly, v.25, n.1, p.1-16, March 2001.

CHEN, W.S.; HIRSCHHEIM, R. A paradigmatic and methodological examination of information systems research from 1991 to 2001. Information Systems Journal, v. 14, n.3, p. 197-235, 2004.

CLAVER, E.; GONZALEZ, R.; LOPIS, J. An analysis of research in information systems (19811997). Information e Management, v. 37, n.4, p. 181-195, 1999.

DENZIN, N. K.; LINCOLN, Y. S. O planejamento da pesquisa qualitativa: teorias e abordagens. Porto Alegre: Artmed, 2006.

GIL, A. C. Métodos e técnicas de pesquisa social. 5. ed. São Paulo: Atlas, 1999.

GODOY, A. A pesquisa qualitativa e sua aplicação em administração de empresas. RAE, v.35, n. 2, p. 57-63, 1995.

HAIR, J. JR. et al. Fundamentos de métodos de pesquisa em administração. Porto Alegre: Bookman, 2005.
HOPPEN, N. Sistemas de informação no Brasil: uma análise dos artigos científicos dos anos 90. Revista Contemporânea de Administração, v. 2, n. 3, p. 151-177, 1998.

HOPPEN, N., LAPOINTE, L.; MOREAU, E. Um guia para avaliação de artigos de pesquisa em Sistemas de Informação. Revista de Administração, v. 2, n.2, p. 42-46, 1996.

HOPPEN, N., LAPOINTE, L.; MOREAU, E. Avaliação de artigos de pesquisa em Sistemas de Informação: proposta de um guia. In: ENANPAD, 21., Angra dos Reis. Anais... Angra dos Reis, 1997. CD-ROOM.

HOPPEN, N.; MEIRELLES, F.S. Sistemas de Informação: um panorama da pesquisa científica entre 1990 e 2003. Revista de Administração de Empresas (RAE), v.45, n.1, p. 24-35, jan./mar. 2005.

LUNARDI, G.; RIOS, L.; MAÇADA, A.C.G. Pesquisa em Sistemas de Informação: uma análise a partir dos artigos publicados no Enanpad e nas principais revistas nacionais de Administração. In: ENANPAD, 28., Brasília. Anais... Brasília: 2005. p. 1-16.

MINAYO, M. C.; SANCHES, O. Quantitativoqualitativo: oposição ou complementaridade? Caderno de Saúde Pública, Rio de Janeiro, v.9, n.3, p. 239-262, 1993.

MORGAN, G.; SMIRICH, L. The case for qualitative research. Academy of Management Review, Mississippi, v. 5, n.4, p. 491-500, 1980.

OLIVEIRA, M.; MAÇADA, A.C.G.; GOLDONI, V. Análise da aplicação do método estudo de caso na área de Sistemas de Informação. In: ENANPAD, 29. Salvador, Anais do 30 ENANPAD, 2006.

OLIVEIRA, M.; MAÇADA, A.C.G.; GOLDONI, V. Análise da aplicação do método estudo de caso na área de Sistemas de Informação. In: ENANPAD, 29., Salvador. Anais... Salvador, 2006.

ORLIKOWSKI, W.; BARAUDI, J. Studying information technology in organizations: research approaches and assumptions. Information Systems Research, v. 2, n. 1, p. 1-29, 1991. 
RICHARDSON, R. Pesquisa social: métodos e técnicas. 2. ed. São Paulo: Atlas, 1989.

SAMPAIO, C. H.; PERIN, M. G. Pesquisa científica da área de marketing: uma revisão histórica. RAC, v.10, n.2, p. 179-202, abr./jun. 2006.

TEIXEIRA JÚNIOR, F. Análise dos Métodos de Pesquisa Utilizados em Artigos de Administração da Informação: Levantamento dos Artigos Publicados nos ENANPADs de 1999 a 2001. In: XXVI ENCONTRO DA ASSOCIAÇÃO NACIONAL DOS PROGRAMAS DE PÓS-GRADUAÇÃO ENANPAD, 26, 2002, Salvador. Anais... Salvador: ANPAD, 2001.

TEIXEIRA JÚNIOR, F. Análise dos métodos de pesquisa utilizados em artigos de Administração da Informação: levantamento dos artigos publicados nos ENANPADs de 1999 a 2001. In: ENANPAD, 27., Salvador. Anais... Salvador: AMPAD, 2001. TRIVIÑOS, A.N.S. Introdução à pesquisa em ciências sociais: a pesquisa qualitativa em educação. São Paulo: Atlas, 2007.

TURBAN, E.; RAINER, K.R.; POTTER. R. E. Administração de tecnologia da informação: teoria e prática. Rio de Janeiro: Elsevier, 2005.

VIEIRA, M. Por uma boa pesquisa (qualitativa) em administração. In: VIEIRA, M.; ZOUAIN, D. M. (Orgs.). Pesquisa qualitativa em administração. 2. ed. Rio de Janeiro: FGV, 2004. p. 13-28.

YIN, R.K. Estudo de caso: planejamento e métodos. 3. ed. Porto Alegre: Bookman, 2005.

ZIMMER, M. V.; FERREIRA, L.; HOPPEN, N. Validação e confiabilidade na área de Sistemas de Informação: uma análise dos artigos publicados no Enanpad entre 1998 e 2006. In: ENANPAD, 31., Rio de Janeiro. Anais... Rio de Janeiro: ANPAD, 2007.

WEBER, M. Metodologia das ciências sociais. São Paulo: Cortez, 1993. 